\title{
Atomic-Layer Deposition for Fabricating Capacitive Micromachined Ultrasonic Transducers: Initial Characterization
}

\author{
Lingli Liu${ }^{1}$, Osama M. Mukdadi² ${ }^{2}$ Marie K. Tripp ${ }^{1}$, \\ Cari F. Herrmann ${ }^{1,3}$, Jean R. Hertzberg'1, Steven M. George ${ }^{3,4}$, \\ Victor M. Bright ${ }^{1}$ and Robin Shandas ${ }^{1,5, *}$ \\ ${ }^{1}$ Dept. of Mechanical Engineering, University of Colorado, Boulder, CO, USA, 80309-0427 \\ ${ }^{2}$ Dept. of Mechanical and Aerospace Engineering, West Virginia University, WV, USA, 26506 \\ ${ }^{3}$ Dept. of Chemistry, University of Colorado, Boulder, CO, USA, 80309-0215 \\ ${ }^{4}$ Dept. of Chemical Engineering, University of Colorado, Boulder, CO, USA, 80309-0424 \\ ${ }^{5}$ Dept. of Pediatric Cardiology, The Children’s Hospital, Denver, CO, USA, 80218
}

(Received March 17, 2007; accepted September 13, 2007)

Key words: capacitive micromachined ultrasonic transducers, medical imaging, atomic-layer deposition

The production of ultrathin membranes facilitates the development of miniature capacitive micromachined ultrasonic transducers (CMUTs), which have potential for use in biomedical imaging applications. Here, we introduce a novel process, incorporating atomic-layer deposition (ALD) and diffusion bonding, for the fabrication of CMUTs with ultrathin membranes. In our fabrication procedure, first a $100 \mathrm{~nm} \mathrm{Al}_{2} \mathrm{O}_{3}$ layer is deposited on an upper silicon wafer by ALD. A $500 \mathrm{~nm}$ gold layer is then deposited on the $\mathrm{Al}_{2} \mathrm{O}_{3}$ layer and patterned to create circular cavities. Then the whole structure is transferred to a bottom wafer by diffusion bonding and the upper silicon wafer is etched away to release the $\mathrm{Al}_{2} \mathrm{O}_{3}$ membrane. Finally, another $30 \mathrm{~nm}$ gold layer is deposited on the membrane for wiring and membrane excitation. Initial results show that extremely flat and uniform membranes can be produced using this process with a RMS roughness of less than $3 \AA$. The ALD technique also provides more options for membrane material selection and has the potential to improve transducer reliability. In this study, the ALDfabricated CMUT array is characterized using numerical models. PACS: 43.38.Bs, 43.40.Dx

${ }^{*}$ Corresponding author: e-mail: Robin.Shandas@colorado.edu 


\section{Introduction}

Ultrasonic array transducers show great potential for use in medical applications, such as real-time three-dimensional imaging, high-frequency imaging, and intravascular and intracardiac ultrasonography. However, the ideal fabrication process for manufacturing reliable matrix-array transducers is still under investigation. Several groups have examined the use of surface micromachining and wafer-bonding techniques to create capacitive micromachined ultrasonic transducers (CMUTs). ${ }^{(1-8)}$ CMUTs fabricated by surface micromachining provide several advantages over conventional piezoelectric transducers: broad bandwidth, good sensitivity, temperature compatibility and potential for electronic integration. Although this fabrication method is promising, it is limited in dimensional tolerances, sealing ability and the range of usable materials. Wafer-bonding technology simplifies the CMUT fabrication process by employing a silicon-on-insulator (SOI) wafer to form the membrane, which ensures process repeatability, better thickness uniformity and controllability of the internal stress within the membrane. However, one uncertainty of the wafer-bonding technique is that it relies on the use of a Si membrane, which may be conductive and could increase the parasitic capacitance. ${ }^{(3)}$

In this paper, we introduce atomic-layer-deposition (ALD) technology into the fabrication process of CMUTs to produce transducer membranes with angstrom-level thickness control. The advantages of ALD include the precise control of membrane thickness and the potential to use a large variety of materials. These capabilities are expected to enable the fabrication of transducer membranes with superior operating characteristics. Also, the transducer reliability may potentially be improved by the enhancement of the surface quality of the membrane. However, when compared with most conventional vapor-phase coating processes [e.g., evaporation, sputtering, chemical vapor deposition (CVD), plasma CVD], the growth rate of ALD is relatively slow which may increase the fabrication period.

\section{Fabrication Process}

We have developed a new fabrication process for CMUTs by employing ALD and membrane transfer technology. ALD is a vapor phase, thin film growth technique that can be used to deposit conformal films with angstrom-level thickness control.(9) ALD relies on sequential, self-limiting reactions between gas-phase precursor molecules and a solid surface. The surface is first exposed to reactant $A$, which reacts with all of the initial surface sites. Then, after purging away the gas-phase products and any remaining reactants, the surface is exposed to reactant $\mathrm{B}$. This reaction regenerates the initial surface sites, thus preparing the surface for another exposure of reactant A. This ABAB... cycle is repeated until the desired film thickness is obtained. A wide variety of materials can be deposited by ALD at low temperatures, often below $180^{\circ} \mathrm{C}$ including oxides, nitrides and metals. ${ }^{(10-14)}$ Many ALD deposited materials show promising characteristics; here we used alumina $\left(\mathrm{Al}_{2} \mathrm{O}_{3}\right)$ as the representative ALD material.

We selected ALD deposited $\mathrm{Al}_{2} \mathrm{O}_{3}$ as our initial CMUT membrane material because it has excellent electrical properties, good adhesion to many surfaces, and excellent thermal 
and chemical stability. For example, an $\mathrm{Al}_{2} \mathrm{O}_{3}$ layer can be deposited on a variety of substrates including $\mathrm{Au}, \mathrm{Co}, \mathrm{Cr}, \mathrm{Cu}, \mathrm{Mo}, \mathrm{Ni}, \mathrm{NiFe}, \mathrm{NiMn}, \mathrm{Pt}, \mathrm{PtMn}, \mathrm{Si}$, polymer, stainless steel, $\mathrm{W}$ and $\mathrm{ZnO} .^{(10,15-20)}$ A $100 \mathrm{~nm}$ thick $\mathrm{Al}_{2} \mathrm{O}_{3}$ ALD layer has a dielectric constant of 7.6.(15)

Trimethylaluminum $\left[\mathrm{Al}\left(\mathrm{CH}_{3}\right)_{3}\right.$ ] (TMA) and $\mathrm{H}_{2} \mathrm{O}$ can be employed for $\mathrm{Al}_{2} \mathrm{O}_{3}$ growth by ALD. The binary reaction $2 \mathrm{Al}\left(\mathrm{CH}_{3}\right)_{3}+3 \mathrm{H}_{2} \mathrm{O} \rightarrow \mathrm{Al}_{2} \mathrm{O}_{3}+6 \mathrm{CH}_{4}$ is separated into two self-limiting reactions: ${ }^{(17)}$

$$
\begin{aligned}
& \text { (A) } 2 \mathrm{Al}-\mathrm{OH}^{*}+2 \mathrm{Al}\left(\mathrm{CH}_{3}\right)_{3} \rightarrow 2 \mathrm{Al}-\mathrm{O}-\mathrm{Al}\left(\mathrm{CH}_{3}\right)_{2}{ }^{*}+2 \mathrm{CH}_{4} \\
& \text { (B) } 2 \mathrm{Al}-\mathrm{CH}_{3}{ }^{*}+3 \mathrm{H}_{2} \mathrm{O} \rightarrow \mathrm{Al}_{2} \mathrm{O}_{3}+2 \mathrm{Al}-\mathrm{OH}^{*}+4 \mathrm{CH}_{4} .
\end{aligned}
$$

where the asterisks designate the surface species. The $\mathrm{Al}_{2} \mathrm{O}_{3}$ films are grown on commercially available polished $<100>$-oriented silicon wafers which have a RMS roughness of $<2 \AA$. The deposition is carried out in a viscous-flow reactor (see Fig.1) with an average growth rate of $1.2 \AA$ per $\mathrm{AB}$ cycle at $177^{\circ} \mathrm{C} .{ }^{(21)}$ A typical $\mathrm{AB}$ cycle takes approximately $12 \mathrm{~s}$ at this temperature. The thickness variation of ALD deposited $\mathrm{Al}_{2} \mathrm{O}_{3}$ has been studied by $\mathrm{x}$-ray reflectivity, ellipsometry, profilometry, and atomic force measurement. ${ }^{(15)}$ ALD-deposited $\mathrm{Al}_{2} \mathrm{O}_{3}$ films have remarkable conformity to the substrate, which ensures uniform thickness over the entire film. Scanning electron microscopy (SEM) images, published elsewhere, also show a high level of uniformity in thickness for ALD deposited $\mathrm{Al}_{2} \mathrm{O}_{3}$ films. ${ }^{(22)}$

Additionally, atomic force microscopy (AFM) images of the ALD deposited $\mathrm{Al}_{2} \mathrm{O}_{3}$ films were acquired using ThermoMicroscopes noncontact ultralevers under tapping mode at a scanning frequency of $1 \mathrm{~Hz}$. ${ }^{(23)}$ Surface areas of $2 \mu \mathrm{m} \times 2 \mu \mathrm{m}$ were recorded using 1024 scan lines and 512 pixels per scan line. Figure 2 shows an AFM image

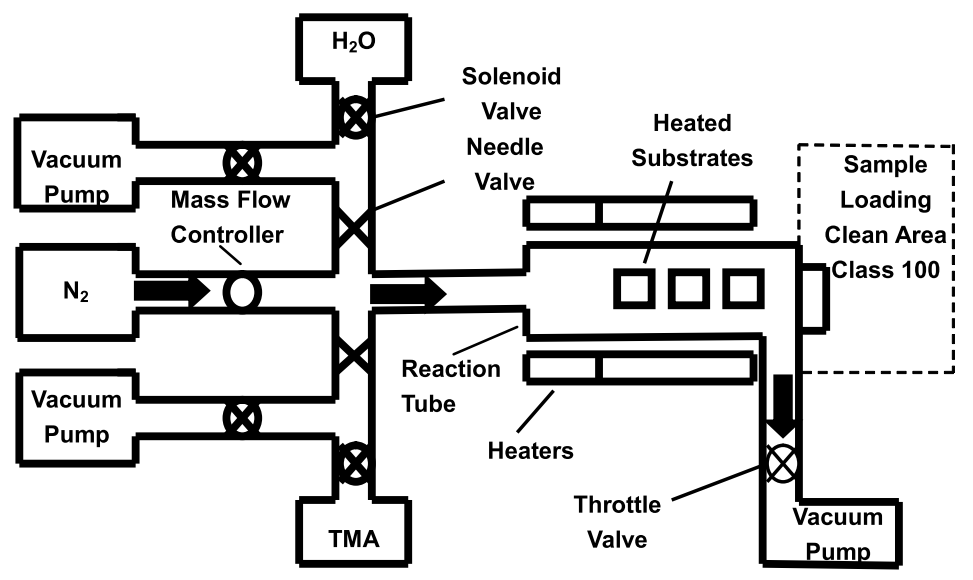

Fig. 1. Schematic diagram of the viscous flow ALD reactor. 


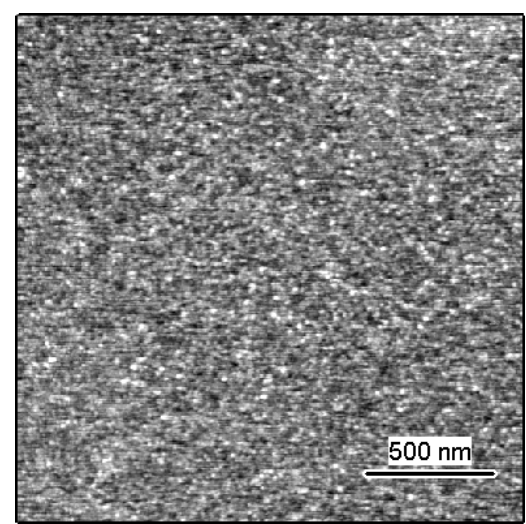

Fig. 2. AFM image of a $100-n m-t h i c k \mathrm{Al}_{2} \mathrm{O}_{3}$ film deposited by ALD. The RMS roughness was determined to be $<3 \AA$.

of a 100 -nm-thick ALD deposited $\mathrm{Al}_{2} \mathrm{O}_{3}$ film, in which the gray scale is chosen such that lighter colors indicate taller surface features. The acquired AFM image was then subjected to a second-order flattening procedure using Autoprobe CP image processing software, and the surface roughness was quantified using the RMS roughness calculated from

$$
R M S=\sqrt{\frac{\sum_{i=1}^{N}\left(z_{i}-z_{a}\right)^{2}}{N-1} .}
$$

where $z_{i}$ is the image height at pixel $i, z_{a}$ is the average height and $N$ is the number of pixels in the AFM image. The results show that the $\mathrm{Al}_{2} \mathrm{O}_{3}$ films are extremely flat and uniform with a RMS roughness of $<3 \AA$. This level of surface quality is one reason why we believe the reliability of CMUTs fabricated using ALD techniques can be improved compared with transducers fabricated using existing techniques.

The CMUT fabrication process consists of 6 steps as outlined in Fig. 3. In step 1, a 100-nm-thick of $\mathrm{Al}_{2} \mathrm{O}_{3}$ is first deposited by ALD on the upper wafer, a polished 1 inch $^{2}$ $<100>$-oriented silicon wafer. The $\mathrm{Al}_{2} \mathrm{O}_{3}$ film thickness is measured using a variableangle spectroscopic ellipsometer (VASE- J.A. Woollam M44). Then we deposit a $500 \pm$ $5 \mathrm{~nm}$ gold layer on the $\mathrm{Al}_{2} \mathrm{O}_{3}$ layer by physical vapor deposition in a vacuum chamber. The $\mathrm{Al}_{2} \mathrm{O}_{3}$ layer will later form the suspended dielectric membrane of the CMUT, and the gold layer will be used as the bonding material during the membrane transfer process in step 4. In step 2, the $500 \pm 5 \mathrm{~nm}$ gold layer is patterned by photolithography and etched through using gold etchant to obtain circular cavity arrays for the transducer. The lateral cavity size is controllable by careful operation of the timed etching; the cavity radius can range from 20 to $100 \mu \mathrm{m}$. In step 3, we prepare the bottom wafer, a polished 1 inch $^{2}<100>$-oriented silicon wafer, by depositing a $0.5 \mu \mathrm{m}$ gold layer and a $2 \mu \mathrm{m}$ 
Step 1

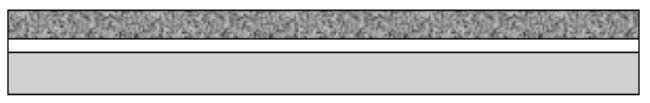

Step 2

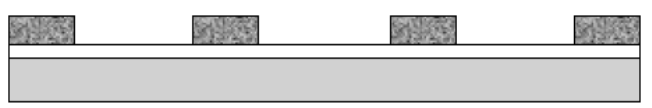

Step 3
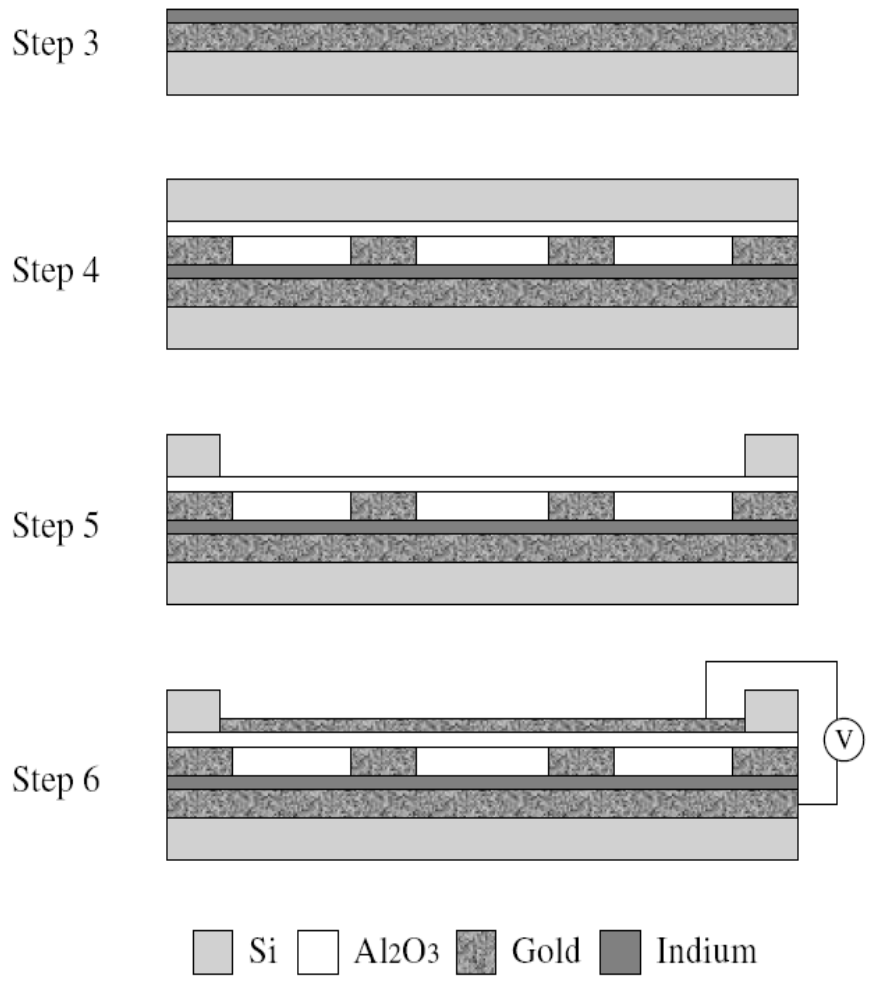

Fig. 3. Fabrication processes of CMUTs by ALD.

indium layer. In step 4, we bond the upper and bottom wafers together in air by goldindium diffusion bonding. Indium is a good material for diffusion bonding because of its cryogenic stability, thermal and electrical conductivity, self-adhesive (ductile) nature and relative ease of application. In step 5, the upper silicon wafer is patterned and etched away using xenon difluoride $\left(\mathrm{XeF}_{2}\right)$ to expose the $\mathrm{Al}_{2} \mathrm{O}_{3}$ layer. ${ }^{(24)}$ Figure 4 shows a picture of a released $\mathrm{Al}_{2} \mathrm{O}_{3}$ membrane array suspended over the transducer cavities formed by gold borders.

Figure 5 shows a picture of a single $\mathrm{Al}_{2} \mathrm{O}_{3}$ membrane from the above array. It has a diameter of $100 \mu \mathrm{m}$, a thickness of $100 \mathrm{~nm}$ and is almost transparent. Finally, in step 6, 


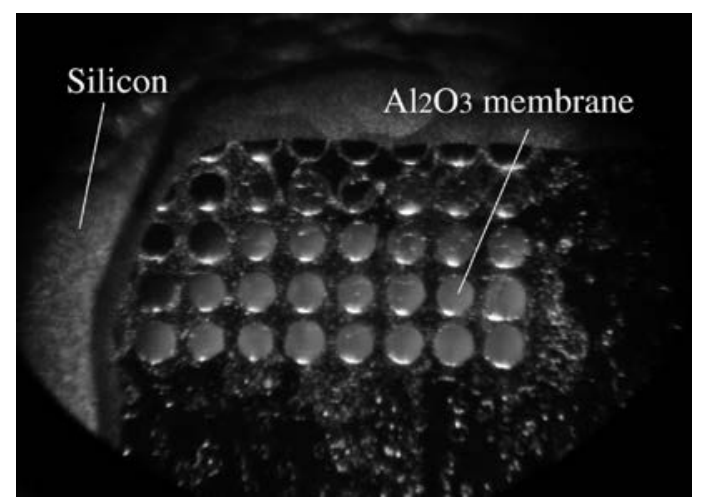

Fig. 4. Picture of a released $\mathrm{Al}_{2} \mathrm{O}_{3}$ membrane array (observed through an optical microscope). The diameter of each membrane is $100 \mu \mathrm{m}$.

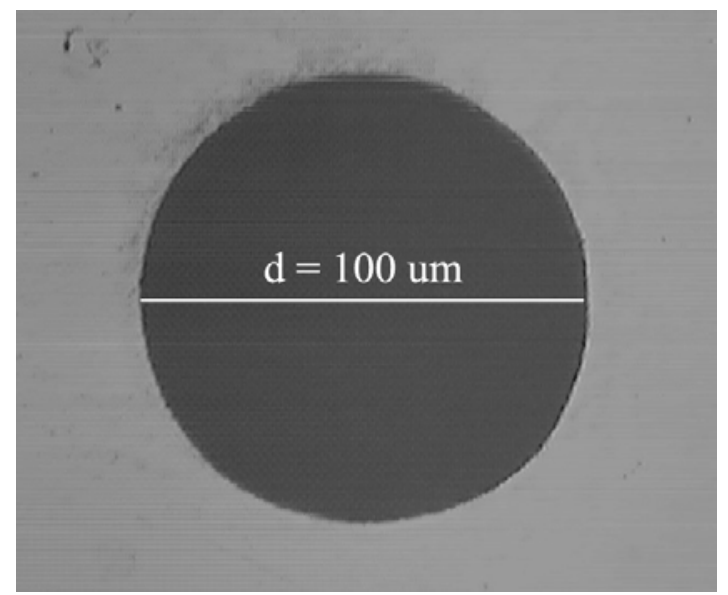

Fig. 5. Image of a single $\mathrm{Al}_{2} \mathrm{O}_{3}$ membrane from the membrane array shown in Fig. 4. The membrane itself is almost transparent, and the deep color within the circular area is the color of the copper substrate of the underlying probe station.

a $30 \pm 5 \mathrm{~nm}$ gold layer is vapor deposited on the $\mathrm{Al}_{2} \mathrm{O}_{3}$ membrane as the upper electrode of the transducer. Now the device is ready for wiring and excitation.

For reliable fabrication and accurate characterization of the transducers, certain issues need further discussion. First, to ensure robust gold-indium diffusion bonding, the thickness of the deposited gold layer should be at least $300 \mathrm{~nm}$. This thickness requirement sets the minimum gap of the device to be $300 \mathrm{~nm}$.

Tensile stress in the membrane will be a factor in the CMUT performance. Usually, the higher the tensile stress, the higher the device resonant frequency and sensitivity will be, but device reliability may decrease. Measurements of tensile stress in 100-nm- 
thick $\mathrm{Al}_{2} \mathrm{O}_{3}$ films deposited by ALD in our laboratory have shown these films to be under a tensile stress of $T=401 \pm 32 \mathrm{MPa} .^{(25)}$ A total of 33 nanofabricated pointer structures $^{(26)}$ were used for this measurement. Pointers from two deposition runs on five separate substrates, with between 1 and 3 pointers on different areas of the substrate, were included in the measurement. Variations in stress across the film and for different deposition runs were less than the measurement uncertainty. The obtained values of stress do not take into account any changes in the overall stress of the membrane due to metallization. Metallization will further change the internal stress within the membrane, depending on the processing temperature, the rate of deposition and the thickness of the deposited metal layer. Physically deposited films can also be under either tensile or compressive stress. ${ }^{(9)}$ The overall stress of the CMUT membrane is determined by both the alumina and the metal layers. More work is needed to quantify the effect of metallization on the overall stress of the CMUT membrane.

\section{Transducer Characterization}

The initial step in evaluating the CMUT performance was through a first-order electromechanical model and equivalent-circuit analysis. In the following simulations, we neglected parasitic loss in the transducer, which stems from membrane heating, acoustic radiation leaking into the supporting structure, and viscous loss in the transducer cavity. We also assume that the driving and receiving electronics can be designed to maximize the power transfer for the transducers of interest.

The equation of motion of a suspended membrane under tension was derived by Mason, ${ }^{(27)}$ and the model was further improved by Ladabaum et al. ${ }^{(1)}$ to calculate the displacement and mechanical impedance of a CMUT membrane as a function of frequency upon excitation by a uniform pressure. We follow the same procedure to numerically characterize the transducers fabricated by ALD.

The model indicates that the variation of the cell size, the membrane thickness and the tensile stress within the membrane will affect the transducer performance. Jin et al. verified the resonant frequency shift of surface-micromachined CMUTs by the change in cell size and membrane thickness. ${ }^{(2)}$ Here we provide further information regarding the changes in transducer performance, including resonant frequencies, transducer transmitting and receiving transfer functions, and changes in internal stresses and cell size. Six transducer types with stress levels from $201 \mathrm{MN} / \mathrm{m}^{2}$ to $401 \mathrm{MN} / \mathrm{m}^{2}$ and cell radii from $20 \mu \mathrm{m}$ to $50 \mu \mathrm{m}$ as listed in Table 1, are used to facilitate the comparison of simulation results. Table 2 lists the symbols that will be used in the following analysis. For $\mathrm{Al}_{2} \mathrm{O}_{3}$ membranes, the Young's modulus $Y_{0}$ is $1.69 \times 10^{11} \mathrm{~Pa}$, Poisson's ratio $\sigma$ is 0.27 , the density $\rho$ is $3270 \mathrm{~kg} / \mathrm{m}^{3}$ and the external uniform pressure $P$ is $1000 \mathrm{~Pa}$. In our numerical model, the membrane thickness $l_{t}$ is $0.1 \mu \mathrm{m}$, the gap thickness $l_{a}$ is $0.5 \mu \mathrm{m}$ and the number of cells in parallel is 1000. When the transducer is operated in air (air-coupled), the acoustic impedance of air is taken as $400 \mathrm{~Pa} \cdot \mathrm{s} / \mathrm{m}$. For immersion applications, the loading acoustic impedance is taken as $1.63 \times 10^{6} \mathrm{~Pa} \cdot \mathrm{s} / \mathrm{m}$, which is equal to the average impedance of human soft tissue.

Figure 6 shows plots of the displacement profile of an air-coupled transducer 
Table 1

Simulation parameters of ALD-fabricated CMUT array.

\begin{tabular}{llllll}
\hline Parameters of CMUT array & & \multicolumn{4}{c}{ Cell radius $\alpha(\mu \mathrm{m})$} \\
\cline { 3 - 5 } & & 20 & 30 & 40 & 50 \\
\hline Tensil force $T\left(\mathrm{MN} / \mathrm{m}^{2}\right)$ & 201 & & & & Type-3 \\
& 301 & & & & Type-2 \\
& 401 & Type-6 & Type-5 & Type-4 & Type-1 \\
\hline
\end{tabular}

Table 2

Nomenclatuire used in numerical analysis of ALD fabricated CMUT array.

\begin{tabular}{lcc}
\hline Symbol & Definition & Value and unit \\
\hline$Y_{0}$ & Young's modulus of membrane & $1.69 \times 10^{11} \mathrm{~Pa}$ \\
$\sigma$ & Poisson's ratio of membrane & 0.27 \\
$\rho$ & Membrane density & $3270 \mathrm{~kg} / \mathrm{m}^{3}$ \\
$P$ & External uniform pressure & $1000 \mathrm{~Pa}$ \\
$l_{t}$ & Membrane thickness & $0.1 \mu \mathrm{m}$ \\
$l_{a}$ & Gap thickness & $0.5 \mu \mathrm{m}$ \\
$n$ & Number of cells in parallel & 1000 \\
$Z_{a}$ & Acoustic impedance & $\mathrm{Pa} \bullet \mathrm{s} / \mathrm{m}$ \\
$\Phi$ & Transformer ratio & $\mathrm{V}$ \\
$V$ & Sinusoidal voltage & $\mathrm{V}$ \\
$I$ & Current & $\mathrm{A}$ \\
$C$ & Static electrical capacitance & $\mathrm{F}$ \\
$Z_{m}$ & Mechanical impedance of membrane & $\mathrm{Pa} \bullet \mathrm{s} / \mathrm{m}$ \\
$F$ & Reaction force of the medium & $\mathrm{N}$ \\
$\bar{V}$ & Spatially averaged velocity of membrane & $\mathrm{m} / \mathrm{s}$ \\
$V_{s}$ & Driving voltage source & $\mathrm{V}$ \\
$R_{s}$ & Internal resistance of $V_{s}$ & $\Omega$ \\
$S$ & Total surface area of transducer & $\mathrm{mm}$ \\
$p_{B}$ & Open-circuit average pressure & $\mathrm{Pa}$ \\
& over the transducer surface & \\
$S_{1}$ & Surface area of a CMUT cell & $\mathrm{mm}{ }^{2}$ \\
\hline
\end{tabular}

membrane (type-1) as a function of frequency under a uniform pressure excitation. It indicates an unloaded resonant frequency of the membrane of $2.2 \mathrm{MHz}$; note, however, that this plot cannot be used to predict the real displacement of the membrane surface since, theoretically, the displacement at the center of the membrane is infinite when it is at resonant frequency. This explains why the maximum simulated displacement, which becomes larger as the mesh grid density of the simulation increases, is larger than the gap thickness. However, when the transducer is operating in real situations, the maximum displacement will be within the gap range because of the load imposed by the medium.

Figure 7 shows the mechanical impedances of the membranes listed in Table 1. It indicates that the smaller the cell radius $a$ and the higher the tensile force $T$, the higher the resonant frequency of the transducer membrane. 


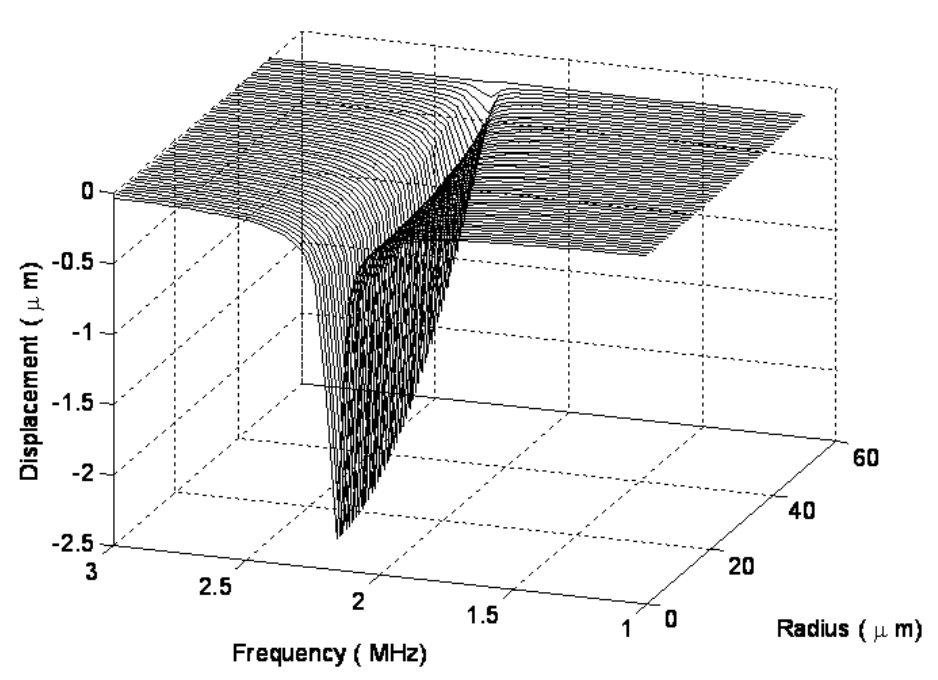

Fig. 6. Displacement profile of the air-coupled transducer membrane (type-1) as a function of frequency excited by a uniform pressure.

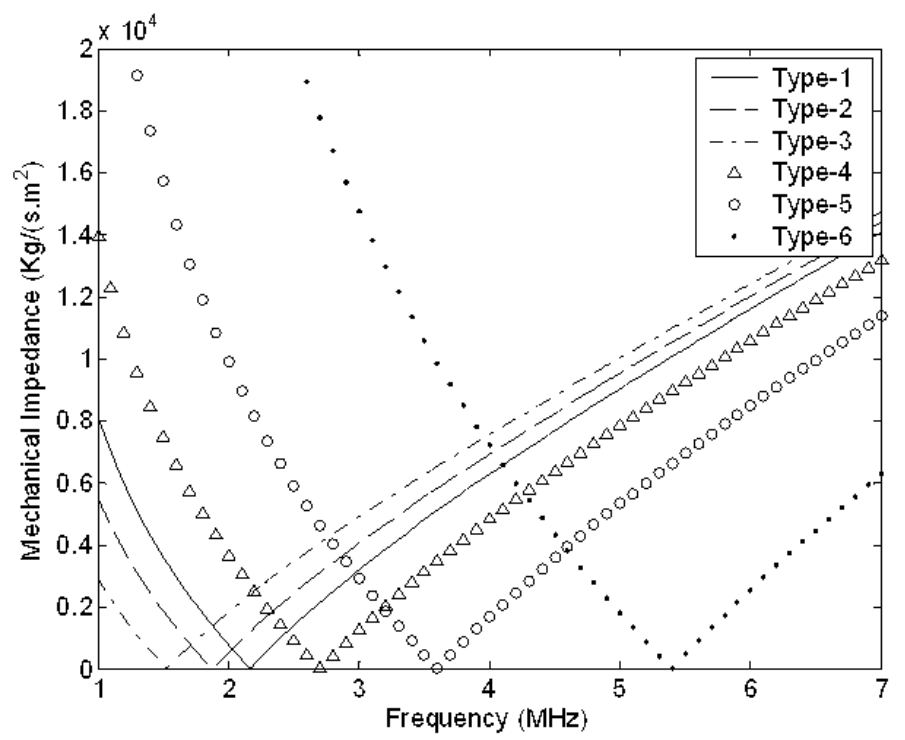

Fig. 7. Mechanical impedances of CMUT transducer membranes.

Note that for ultrathin membranes with very small gap widths, the application of high bias voltages can cause the membrane to collapse onto the substrate. Here, we analyze the electrostatic collapse voltage through the lumped electromechanical model, assuming that the membrane's restoring force is a linear function of its displacement, and neglecting all electrical fringing fields and membrane curvature. 
The lumped electromechanical model consists of a linear spring, a mass and a parallel-plate capacitor. ${ }^{(1,5)}$ The electrical capacitance for a parallel plate capacitor is:

$$
C(t)=\frac{\varepsilon_{0} \varepsilon S_{1}}{\varepsilon_{0} l_{t}+\varepsilon l_{a}(t)}=\frac{\varepsilon_{0} \varepsilon S_{1}}{\varepsilon_{0} l_{t}+\varepsilon\left(l_{a 0}-x\right)}
$$

where $l_{t}$ is the membrane thickness, $l_{a 0}$ is the original gap distance, $x$ is the membrane displacement, $S_{1}$ is the surface area of a CMUT cell, $\varepsilon_{0}$ is the dielectric constant of air and $\varepsilon$ is the dielectric constant of the membrane material. When applying a DC voltage between the membrane and the substrate, the electrostatic attraction force on the membrane is

$$
F_{E}=-\frac{d}{d x}\left(\frac{1}{2} C(t) V_{D C}^{2}\right)=\frac{\varepsilon_{0} \varepsilon^{2} S_{1} V_{D C}^{2}}{2\left(\varepsilon_{0} l_{t}+\varepsilon l_{a 0}\right)^{2}}
$$

The total restoring spring force is

$$
F_{S}=k x
$$

where $k$ is the spring constant of the membrane, which is defined as the ratio of the pressure to the volume displacement: ${ }^{(27)}$

$$
k=\frac{T S_{1} l_{t}}{\left(\frac{c}{d}-\frac{a}{2} \frac{J_{0}(a \sqrt{d / c})}{J_{1}(a \sqrt{d / c})} \sqrt{\frac{c}{d}}+\frac{a^{2}}{8}\right)} .
$$

where

$$
c=\frac{\left(Y_{0}+T\right) l_{t}^{2}}{12 \rho\left(1-\sigma^{2}\right)}, d=\frac{T}{\rho} .
$$

and $T, Y_{0}, \rho, \sigma$ and $a$ are the internal stress, Young's modulus, density, Poisson's ratio and the diameter of the membrane, respectively. $J_{0}\left(\right.$ ) and $J_{1}($ ) are the zeroth-and first-order Bessel functions of the first kind, respectively.

By equating $F_{E}$ to $F_{S}$, the voltage required to keep the membrane at certain displacement $x$ can be solved. As $V_{D C}$ is increased, there is a point at which $\partial V_{D C} \partial x=0$, and the electrostatic force overwhelms the restoring spring force, leading to the collapse of the membrane. At the collapse point

$$
x=\frac{1}{3}\left(l_{a 0}+\frac{\varepsilon_{0}}{\varepsilon} l_{t}\right) .
$$


and the corresponding collapse voltage is

$$
V_{\text {collapse }}=\sqrt{\frac{8 k\left(l_{a 0}+\frac{\varepsilon_{0}}{\varepsilon} l_{t}\right)^{3}}{27 S_{1} \varepsilon_{0}}} .
$$

A recently developed electrical-equivalent circuit model ${ }^{(1,4)}$ is employed to find the electrical impedance of the ALD-fabricated CMUTs based on the characteristic dimensions obtained from our first-level fabrication process, see Fig. 8. In this model, the linear operation of the electrostatic cells is described by means of a two-port network with a mechanical and an electrical port. In the electrical port, the static electrical capacitance of each cell can be calculated by eq. (2). When a voltage $V=V_{D C}+V_{a c}$ $\sin (\omega t)$ is applied to the capacitor, where $V_{D C}$ is the bias voltage and $V_{a c}<<V_{D C}$ is a smallsignal AC voltage, the current flowing through the device is

$$
I=\frac{d}{d t} Q=\frac{d}{d t}(C(t) V(t))=C(t) \frac{d}{d t} V(t)+V(t) \frac{d}{d t} C(t) .
$$

We assume that $C(t)=C_{0}+C_{a c} \sin (\omega t+\phi)$ where $C_{a c}<<C_{0}$ since a small-signal AC voltage is applied. Then the current becomes

$$
I=C_{0} \frac{d}{d t} V_{a c}(t)+V_{D C} \frac{d}{d t} C_{a c}(t)
$$
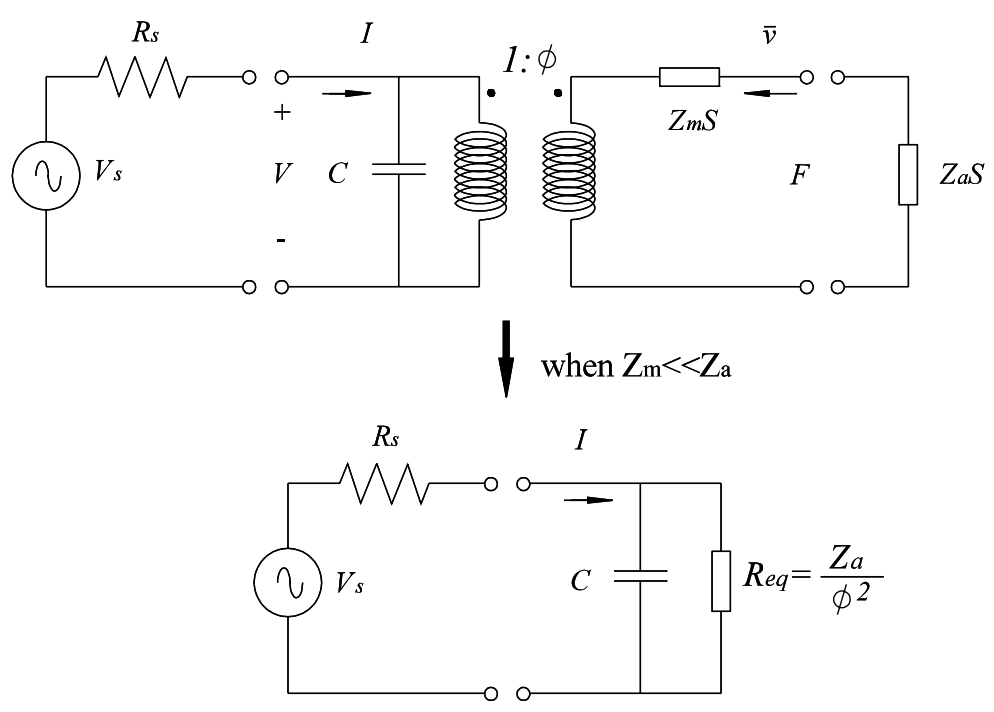

Fig. 8. Transducer electrical-equivalent circuit model. 
By differentiating eq. (2) we obtain:

$$
\frac{d}{d t} C_{a c}(t)=-\frac{\varepsilon_{0} \varepsilon^{2} S_{1}}{\left(\varepsilon_{0} l_{t}+\varepsilon l_{a 0}\right)^{2}} \frac{d}{d t} l_{a}(t) .
$$

The derivative of the gap thickness is equal to the velocity of the membrane $\bar{v}=\frac{d}{d t} l_{a}(t)$, so that

$$
I=C_{0} \frac{d}{d t} V_{a c}(t)-\frac{V_{D C} \varepsilon_{0} \varepsilon^{2} S_{1}}{\left(\varepsilon_{0} l_{t}+\varepsilon l_{a 0}\right)^{2}} \bar{v}
$$

In eq. (11), the average membrane velocity in the mechanical port is related to the current and voltage in the electrical port. The transformer ratio is thus defined as

$$
\phi=\frac{V_{D C} \varepsilon_{0} \varepsilon^{2} S_{1}}{\left(\varepsilon_{0} l_{t}+\varepsilon l_{a 0}\right)^{2}} .
$$

and the current can be written as the sum of the electrical and mechanical components

$$
I=C_{0} \frac{d}{d t} V_{a c}(t)-\phi \bar{v}
$$

The electromechanical transformer ratio $\phi$ determines the efficiency of the CMUT and is a function of the DC bias voltage and the various thicknesses and dielectric properties of the layered materials. In our simulation, the bias voltage is chosen to be $80 \%$ of the collapse voltage. Using the type- 1 transducer membrane for example, the bias voltage is calculated to be $19 \mathrm{~V}$ and the device capacitance is $135 \mathrm{pF}$. The bias voltage indicates a low power requirement for the transducer, and it also implies that this type of transducer may be limited to small-depth imaging applications such as intravascular, epicardial, and laparoscopic applications, since the acoustic pressure generated by the transducer will be relatively low and may be unable to penetrate deep into tissue. Figure 9 shows the simulation results of electrical input impedance for the type- 1 transducer under both aircoupled and immersion conditions. The real part of the immersion transducer impedance is much lower than that of the air-coupled transducer. The high imaginary-to-real impedance ratio leads to an impedance mismatch between the transmitting and receiving electronics and may decrease the dynamic range of the immersion transducer.

The transmitting transfer function (TTF) and receiving transfer function (RTF) describe the sensitivity characteristics of the transducer. The derivation of these transfer functions begins with the canonical equations of a single cell: ${ }^{(4,28)}$ 


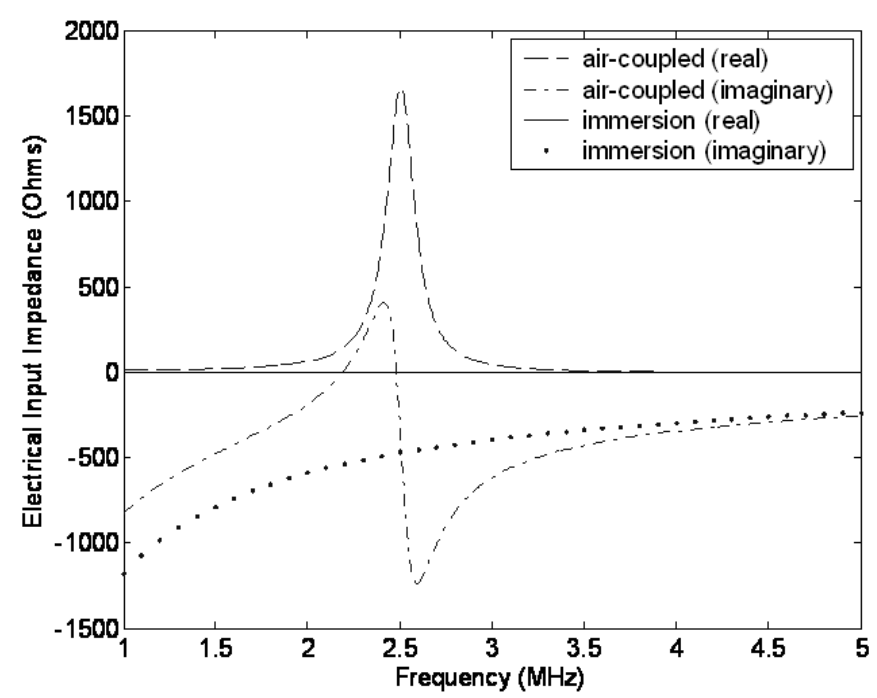

Fig. 9. Electrical input impedance of type-1 CMUT array.

$$
V=\frac{I}{j \omega C}+\frac{\phi \bar{v}}{j \omega C}, \quad F=\frac{\phi I}{j \omega C}+\left(Z_{m}+\frac{\phi^{2}}{j \omega C}\right) \bar{v}
$$

where $V$ is the sinusoidal voltage (which could be both the applied or the generated voltage), $I$ is the current, $\omega$ is the angular frequency, $C$ is the static electrical capacitance of each cell, $Z_{m}$ is the mechanical impedance of each membrane, $F$ is the reaction force of the medium on the vibrating surface, and $\bar{v}$ is the spatially averaged velocity of the membrane.

For a transducer array consisting of $n$ cells connected in parallel, the active static capacitance is $n C$, the overall transformer ratio is $n \phi$ and the total mechanical impedance of the array of membranes is $n Z_{m}$. We can extend the canonical equations of a single cell to those of a transducer array:

$$
V=\frac{I}{j n \omega C}+\frac{\phi \bar{v}}{j \omega C}, \quad F=\frac{n \phi I}{j n \omega C}+\left(n Z_{m}+\frac{n \phi^{2}}{j \omega C}\right) \bar{v}
$$

When the transducer is driven by a voltage source $V_{s}$ whose internal resistance is $R_{s}$, the applied sinusoidal voltage is $V$. The motion of the membranes is produced by an apparent force $-\phi V$ acting on the total mechanical impedance $Z_{m}+Z_{a}$, and the force transmitted to the medium $F$ is related to the voltage source $V_{s}$ by means of the TTF: 


$$
T T F=-\frac{F}{V_{s}}=\frac{n \phi}{\left(1+j n \omega C R_{s}\right)\left(1+\frac{Z_{m}}{Z_{a}}\right)+n \phi^{2} \frac{R_{s}}{Z_{a} S}}
$$

where $Z_{m}$ is the mechanical impedance of the membrane and $Z_{a}$ is the specific acoustic impedance of the medium $\left(Z_{a}=\rho c\right.$, where $\rho$ is the density of the medium, and $c$ is the propagation speed of sound in that medium). For example, the acoustic impedance for air is $400 \mathrm{~Pa} \cdot \mathrm{s} / \mathrm{m}$ and average impedance of human soft tissue is $1.63 \times 10^{6} \mathrm{~Pa} \cdot \mathrm{s} / \mathrm{m}$.

For immersion applications, if we let $R_{s}$ be small, the TTF approaches the transformer ratio $\phi$, since in most immersion applications, the acoustic impedance of the immersion medium, $Z_{a}$, is much greater than the membrane impedance, $Z_{m}$, and the equivalent circuit can be simplified by neglecting $Z_{m}$, as shown in Fig. 8 .

Figures 10-13 show plots of TTFs versus excitation frequency for air-coupled and immersion transducers with different cell sizes and tensile stresses. Note that the TTF is largely independent of frequency for immersion application, and the efficiency remains of the same order of magnitude. This indicates that the larger the cell size and the higher the tensile stress, the higher the transmitting transfer ability of the transducers. The model also predicts that the transmitting transfer ability will reach its minimum if the cell radius is less than $30 \mu \mathrm{m}$.

When the transducer is used as a receiver, the incident pressure field is represented by a force generator $n p_{B} S$, where $S$ is the total surface area of the transducer and $p_{B}$ is the average pressure over the surface of the transducers when the membranes are blocked (i.e.,

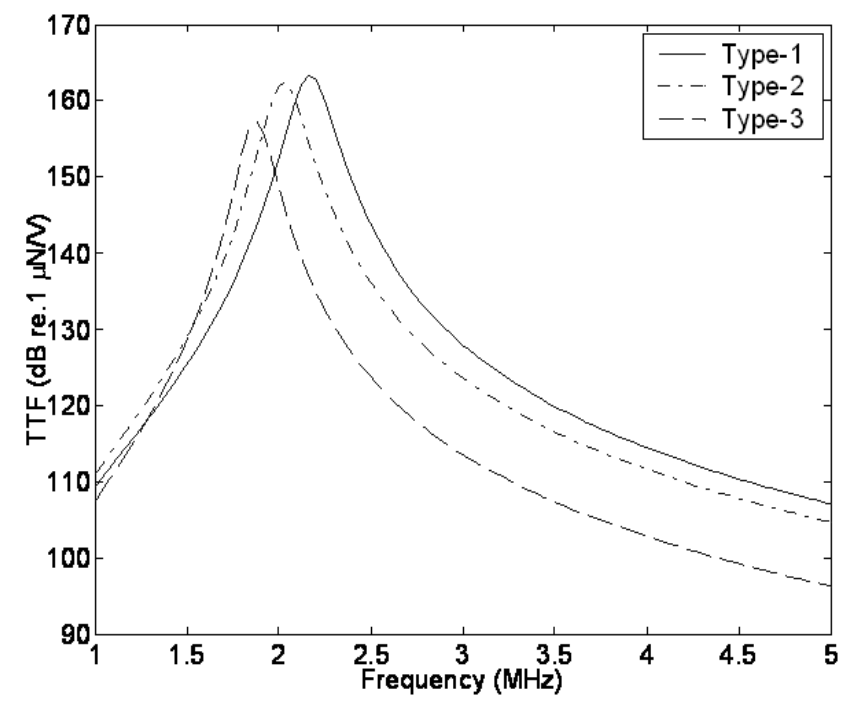

Fig. 10. TTF versus excitation frequency for air-coupled transducers with $a=50 \mu \mathrm{m}$. 


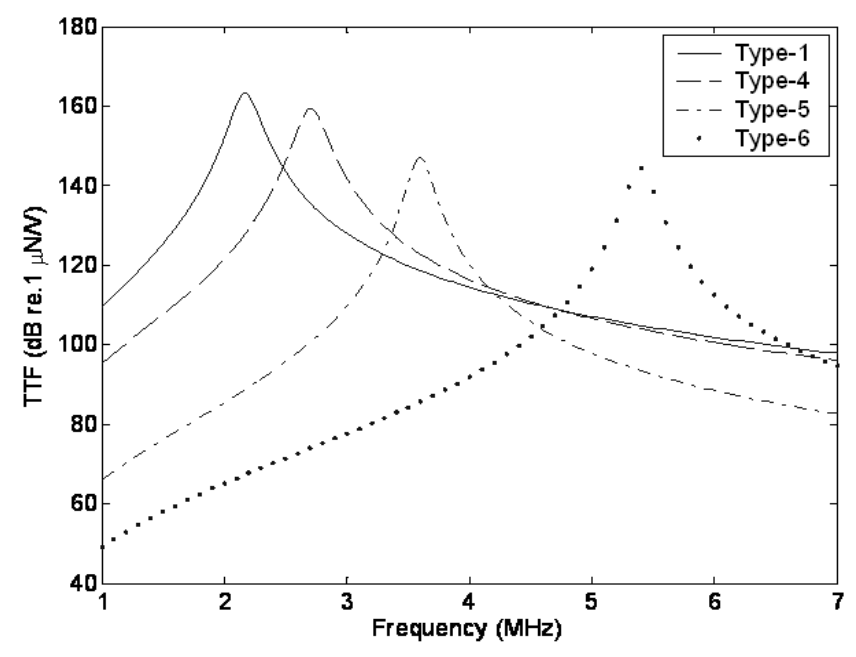

Fig. 11. TTF versus excitation frequency for air-coupled transducers with $T=401 \mathrm{MN} / \mathrm{m}^{2}$.

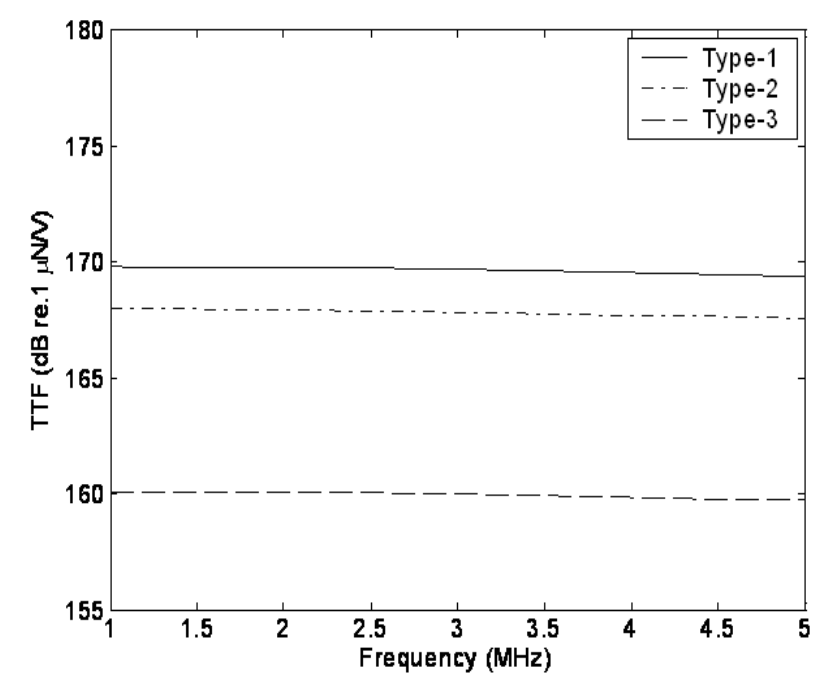

Fig. 12. TTF versus excitation frequency for immersion transducers with $a=50 \mu \mathrm{m}$.

the circuit is open). The RTF is defined as the open-circuit output voltage divided by the average active force: ${ }^{(4)}$

$$
R T F=\frac{V_{0}}{n p_{B} S}=\frac{\phi}{\phi^{2}+j n^{2} \omega C S\left(Z_{m}+Z_{a}\right)} .
$$




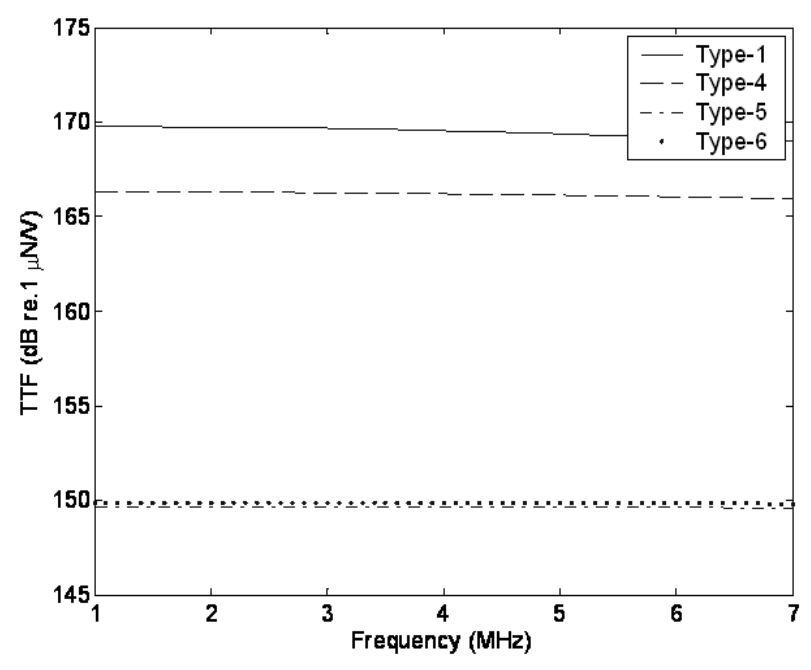

Fig. 13. TTF versus excitation frequency for immersion transducers with $T=401 \mathrm{MN} / \mathrm{m}^{2}$.

Figures 14-17 plot the receiving transfer functions versus exciting frequency for aircoupled and immersion transducers with different cell sizes and tensile stresses. The figures indicate that for transducers with the same cell size, the higher the tensile stress, the higher the receiving sensitivity; while for the transducers with the same tensile stress, the smaller the cell size, the higher the receiving sensitivity. Also, we observe that the sensitivity curves for the immersion transducers decrease monotonically because the immersion transducers do not operate in the resonant mode. This has been demonstrated experimentally(6) and implies that immersion transducers have a broader bandwidth than air-coupled transducers. The bandwidths (both transmitting and receiving) of the aircoupled transducers are dominated by the mechanical impedance of the membrane, and we expect resonance in accord with the results shown in Fig. 7. On the other hand, the bandwidths of the immersion transducers are determined by the transformer ratio. However, the immersion transducers are much less sensitive overall, particularly at high frequencies, which may limit their utility for high sensitivity applications. The simulation results also indicate that to optimize the sensitivity of ALD-fabricated CMUTs, both the dimension (cell size) and the stress need to be considered.

\section{Conclusions}

A new method, using ALD technology, has been proposed for fabricating CMUT arrays. ALD offers many advantages over conventionally micromachined CMUTs, including the precise control of membrane thickness, lower cost due to a reduction in the 


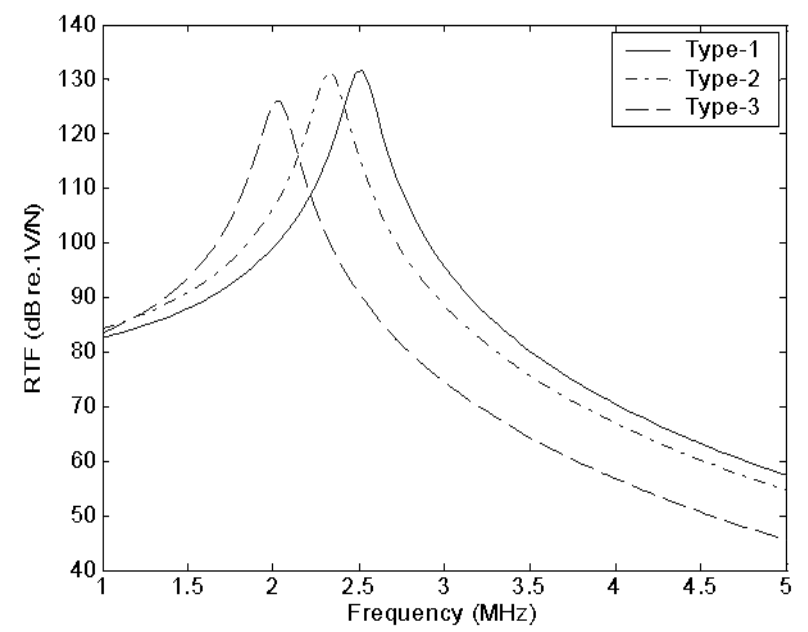

Fig. 14. RTF versus excitation frequency for air-coupled transducers with $a=50 \mu \mathrm{m}$.

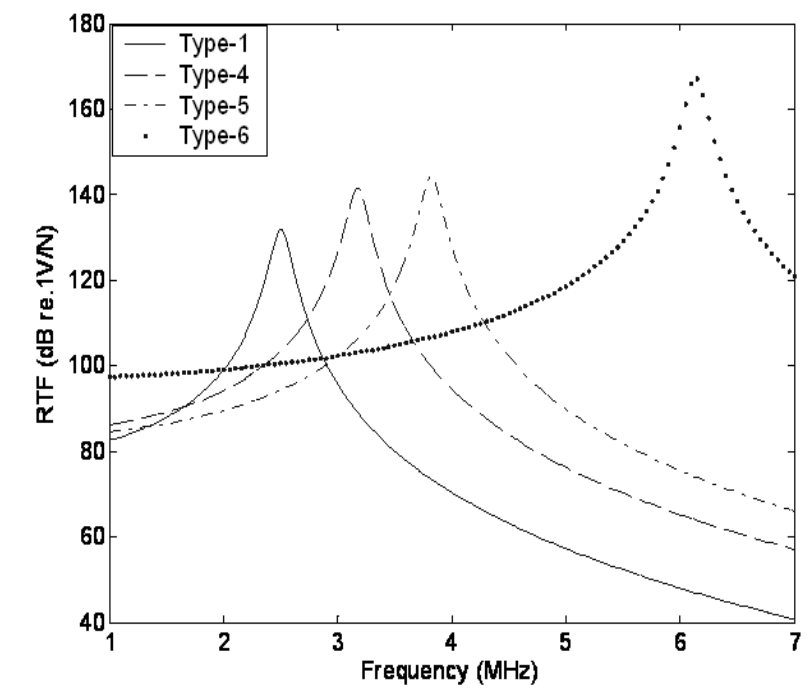

Fig. 15. RTF versus excitation frequency for air-coupled transducers with $T=401 \mathrm{MN} / \mathrm{m}^{2}$.

number of fabrication steps, the potential to use a large variety of materials and increased reliability due to the enhanced surface quality of the membranes. ALD-fabricated CMUT arrays were also characterized by numerical simulations. The simulation results indicate that transducer performance characteristics, such as resonant frequency, TTF and RTF, are affected by the cell size and stress within the membrane: the smaller the cell radius 


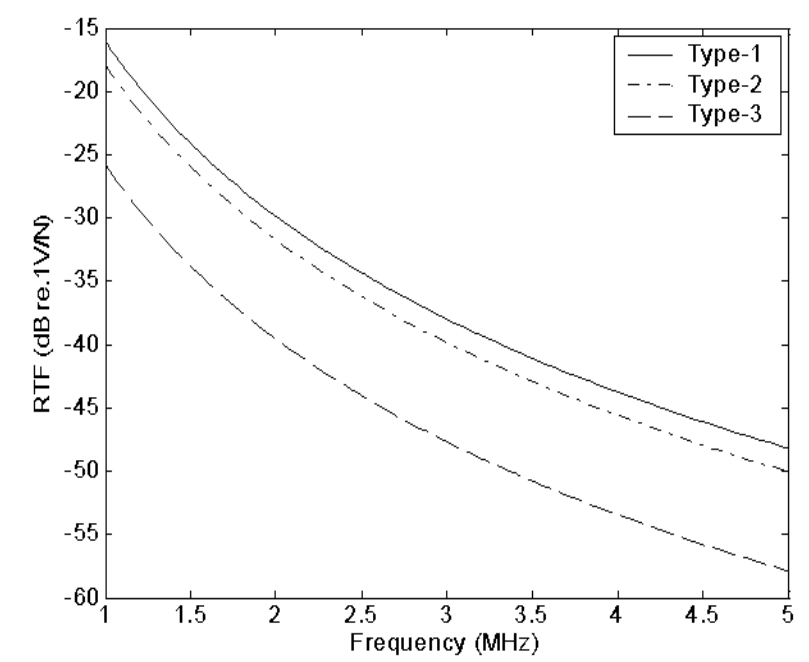

Fig. 16. RTF versus excitation frequency for immersion transducers with $a=50 \mu \mathrm{m}$.

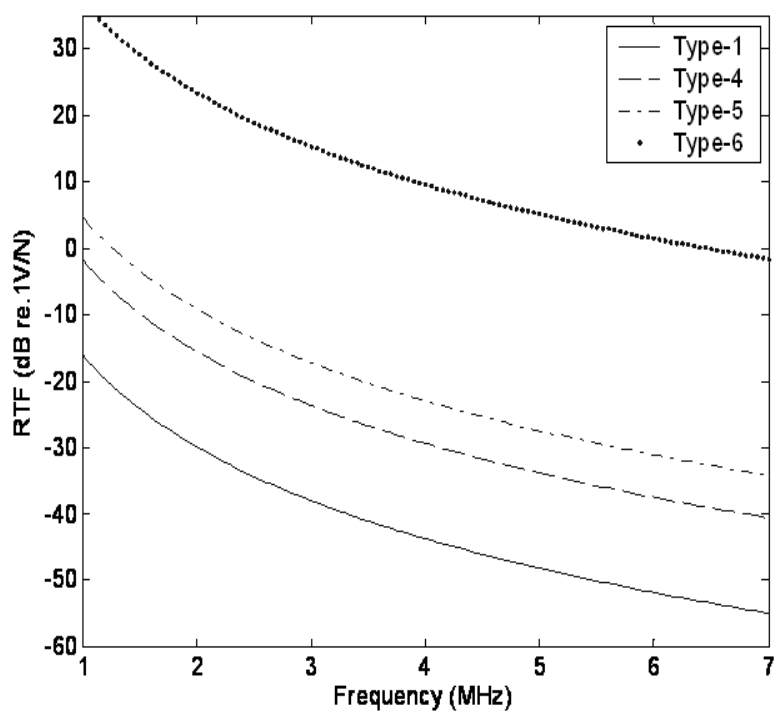

Fig. 17. RTF versus excitation frequency for immersion transducers with $T=401 \mathrm{MN} / \mathrm{m}^{2}$.

and the higher the tensile force, the higher the resonant frequency; and the larger the cell size and the higher the tensile stress, the higher the transmitting transfer ability. For transducers with the same cell size, the higher the tensile stress, the higher the receiving sensitivity; while for transducers with the same tensile stress, the smaller the cell size, the higher the receiving sensitivity. These results may help us identify design goals to improve the transducer performance. 


\section{Acknowledgements}

This work was supported in part by grants from NSF (EECS - 0225405, CTS 0421461) and NIH (HL 072738 and HL 079868). The authors also appreciate Professor Y.C. Lee from the Department of Mechanical Engineering, Professor Z. Popovic from the Department of Electrical and Computer Engineering for providing some of the experimental devices, Dr. R. A. Saravanan and graduate student Y. J. Chang and A. D. Laws from the Department of Mechanical Engineering for providing experimental training, and R. A. Wind from the Department of Chemistry, University of Colorado for the AFM image.

\section{References}

1. I. Ladabaum, X. C. Jin, H. T. Soh, A. Atalar and B. T. Khuri-Yakub: IEEE Trans. Ultrason. Ferroelectri. Freq. Control 45 (1998) 678.

2. X. C. Jin, I. Ladabaum and B. T. Khuri-Yakub: J. Microelectromechanical Syst. 7 (1998) 295.

3. Y. L. Huang, A. S. Ergun, E. Haeggstrom, M. H. Badi and B. T. Khuri-Yakub: J. Microelectromechanical Syst. 12 (2003) 128.

4. A. Caronti, G. Caliano, A. Iula and M. Pappalardo: IEEE Trans. Ultrason. Ferroelectri. Freq. Control 49 (2002) 159.

5. A. Bozkurt, I. Ladabaum, A. Atalar and B. T. Khuri-Yakub: IEEE Trans. Ultrason. Ferroelectri. Freq. Control 46 (1999) 1364.

6. X. C. Jin, O. Oralkan, F. L. Degertekin and B. T. Khuri-Yakub: IEEE Trans. Ultrason. Ferroelectri. Freq. Control 48 (2001) 750.

7. G. Caliano, R. Carotenuto, A. Caronti and M. Pappalardo: IEEE Ultrasonics Symp. (Munich, 2002) p. 1067.

8. M. Pappalardo, G. Caliano, A. Caronti, F. D’Alessio, C. Cucco, E. Cianci and V. Foglietti: IEEE Symp. on Ultrasonics (Hawaii, 2003) p. 1955.

9. H. S. Nalwa: Handbook of Thin Film Materials (Academic Press, San Diego, 2002).

10. M. Leskela and M. Ritala: Thin Solid Films 409 (2002) 138.

11. J. D. Ferguson, A. W. Weimer and S. M. George: Thin Solid Films 413 (2002) 16.

12. J. D. Ferguson, E. R. Smith, A. W. Weimer and S. M. George: J. Electrochem. Soc. 151 (2004) G528.

13. B. S. Lim, A. Rahtu and R. G. Gordon: Nature Mater. 2 (2003) 749.

14. J. D. Ferguson, A. R. Yoder, A. W. Weimer and S. M. George: Appl. Surf. Sci. 226 (2004) 393.

15. M. D. Groner, J. W. Elam, F. H. Fabreguette and S. M. George: Thin Solid Films 413 (2002) 186.

16. X. H. Liang, L. F. Hakim, G. D. Zhan, J. A. McCormick, S. M. George, A. W. Weimer, J. A. Spencer, K. J. Buechler, J. Blackson, C. J. Wood and J. R. Dorgan: J. Am. Ceram. Soc. 90 (2007) 57.

17. A. W. Ott, J. W. Klaus, J. M. Johnson and S. M. George: Thin Solid Films 292 (1997) 135.

18. L. F. Hakim, J. A. McCormick, G. D. Zhan, A. W. Weimer, P. Li and S. M. George: J. Am. Ceram. Soc. 89 (2006) 3070.

19. H. L. Lu, Y. B. Li, M. Xu, S. J. Ding, L. Sun, W. Zhang and L. K. Wang: Chin. Phys. Lett. 23 (2006) 1929.

20. R. K. Grubbs, C. E. Nelson, N. J. Steinmetz and S. M. George: Thin Solid Films 467 (2004) 16. 
21. J. W. Elam, M. D. Groner and S. M. George: Rev. Sci. Instrum. 73 (2002) 2981.

22. N. D. Hoivik, J. W. Elam, R. J. Linderman, V. M. Bright, S. M. George and Y. C. Lee: Sens. Actuators A 103 (2003) 100.

23. J. W. Elam, Z. A. Sechrist and S. M. George: Thin Solid Films 414 (2002) 43.

24. L.-A. Liew: Polymer-Derived Silicon Carbonitride As a New Technology for Microelectromechanical Systems (Ph.D. thesis, University of Colorado, Boulder, 2002).

25. M. K. Tripp, C. Stampfer, C. F. Herrmann, C. Hierold, S. M. George and V. M. Bright: 13th Int. Conf. Solid-State Sensors, Actuators, and Microsystems (Seoul, 2005) p.851.

26. B. P. Vandrieenhuizen, J. F. L. Goosen, P. J. French and R. F. Wolffenbuttel: Sens. Actuators A 37-8 (1993) 756.

27. W. P. Mason: Electromechanical Transducers And Wave Filters (D. Van Nostrand Co., New York, 1948).

28. L. E. Kingsler, A. R. Frey, A. B. Coppens and J. V. Sanders: Fundamentals of Acoustics (John Wiley \& Sons, New York, 1982). 